\title{
Metachronous Skin Metastasis of Gastric Cancer: Case Report
}

\author{
${ }^{1}$ Department of surgery, Lebanese University, Lebanon \\ ${ }^{2}$ Medical student, Lebanese University, Lebanon \\ ${ }^{3}$ Medical student, Lebanese American University, Lebanon \\ ${ }^{4}$ Department of surgery, Mount Lebanon Hospital, Lebanon
}

Mohamad Rakka ${ }^{*}$, Nagham Bazzi ${ }^{2}$, Alaa Haidar ${ }^{1}$, Ali El Safadi ${ }^{3}$ and Georges Chahine ${ }^{4}$

Submission: September 09, 2019; Published: October 10, 2019

*Corresponding author: Mohamad Rakka, General Surgery Resident, Department of Surgery, Lebanese University, Faculty of Medicine, Beirut, Lebanon

\section{Abstract}

An 86- year- old man, had undergone gastric surgery for peptic ulcer disease 40 years ago and he was diagnosed with gastric adenocarcinoma. Then he underwent a subtotal gastrectomy with regional lymphadenectomy, followed by Roux en Y gastrojejunostomy, and adjuvant chemotherapy. One and a half years later, the patient presented with a solid mass on his abdominal wall. Complete excision of the mass revealed a moderately differentiated adenocarcinoma consistent with the primary tumor of the stomach followed by fluorodeoxyglucose positron emission tomography (FDG PET) scan that showed hypermetabolic activity in the liver and the para-aortic lymph nodes, suggestive of distant metastasis.

Keywords: Gastric cancer; Metachronous skin metastasis; Chemotherapy for gastric cancer

\section{Introduction}

Gastric cancer is the fifth most common cancer worldwide but the third leading cause of cancer related deaths according to the World Health Organization (WHO). Early symptoms include heartburn, persistent indigestion and difficulty swallowing. Outcomes are usually poor with $10 \%$ five-year survival [1] Gastric cancer usually gives metastasis to the liver, peritoneal cavity and lymph nodes and rarely to the skin [1]. Generally, skin metastasis from gastric are inoperable and are associated with an advanced stage of the disease. In this article, we report a rare case of metachronous skin metastasis of gastric adenocarcinoma, and we discuss the presentation, diagnosis and management of this rare metastasis. We are reporting a rare case of skin metastasis of gastric cancer in an 86-year-old male, first case reported in Lebanon.

\section{Case Presentation}

This is a case of an 86-year-old man, nonsmoker, nonalcoholic, with no known food and drug allergy, had undergone gastric surgery for peptic ulcer disease 40 years ago. He has an anemia that was discovered one year ago and since then he has been taking iron supplements. The patient didn't undergo an esophagogastroduodenoscopy before.

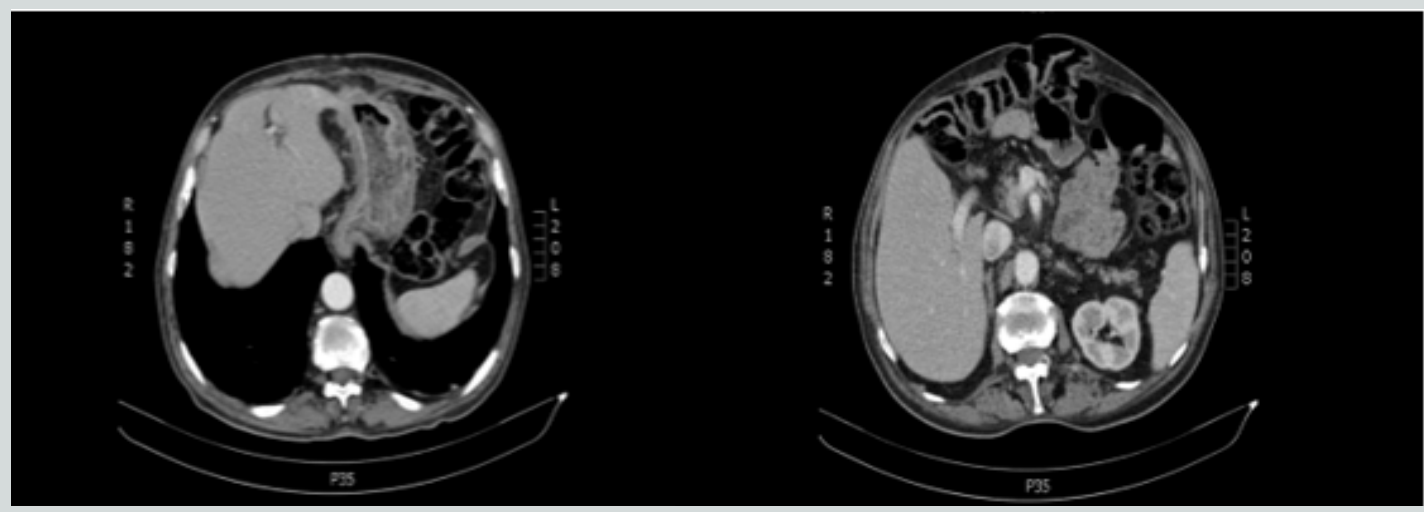

Figure 1: CT scan of the abdomen with IV contrast revealed diffuse parietal thickening of the stomach mainly at the level of the antrum and a supra-umbilical midline abdominal wall hernia containing a small segment of the transverse colon. 


\section{Juniper Online Journal of Case Studies}

He presented with abdominal wall bulge. On physical examination, the vital signs were within normal range. The abdomen was soft, there was a reducible incisional hernia at the site of previous laparotomy incision without sings of incarceration or strangulation. No other abnormal findings. Laboratory tests were within normal range except for anemia (Hemoglobin: 8g/dl, Hematocrit: 27\%). A computerized tomography (CT) scan of the abdomen with intravenous contrast revealed diffuse parietal thickening of the stomach mainly at the level of the antrum and a supra-umbilical midline abdominal wall hernia containing a small segment of the transverse colon as seen in Figure 1. Esophagogastroduodenoscopy was done and showed a $2 \mathrm{~cm}$ vegetating and necrotic tumor in the antrum. A Biopsy was taken. The histology revealed a well to moderately differentiated adenocarcinoma.

FDG PET/CT was done and showed hypermetabolic activity in the stomach with no distant metastasis. Thus, the patient was planned for total gastrectomy, regional lymphadenectomy and incisional hernia repair. Generalized abdominal exploration revealed no ascites, no liver metastasis and no peritoneal carcinomatosis. A loop gastrojejunostomy was identified, in addition, a large tumor was palpated in the antrum that was invaded the anastomosis and the transverse colon.

A subtotal gastrectomy, resection of the previous gastrojejunostomy, and segmental resection of the transverse colon, followed by Roux en Y gastrojejunostomy and colo-colic anastomosis where performed. The hernia defect was then repaired. Patient's postoperative course was uneventful, he was discharged home on the 6th day postoperative day in a good condition.

The pathology study revealed a well to moderately differentiated adenocarcinoma, intestinal type, infiltrating the gastric wall up to the sub serosa $\mathrm{T}=6.5 \mathrm{~cm}$. The gastric, jejunal and colonic surgical limits were free (R0). There was one positive lymph node (1/12) staining pT4N1M0. Immunohistochemistry test showed negative MSI and PD-L1. The patient was started then on adjuvant chemotherapy: Capecitabine per os twice daily.

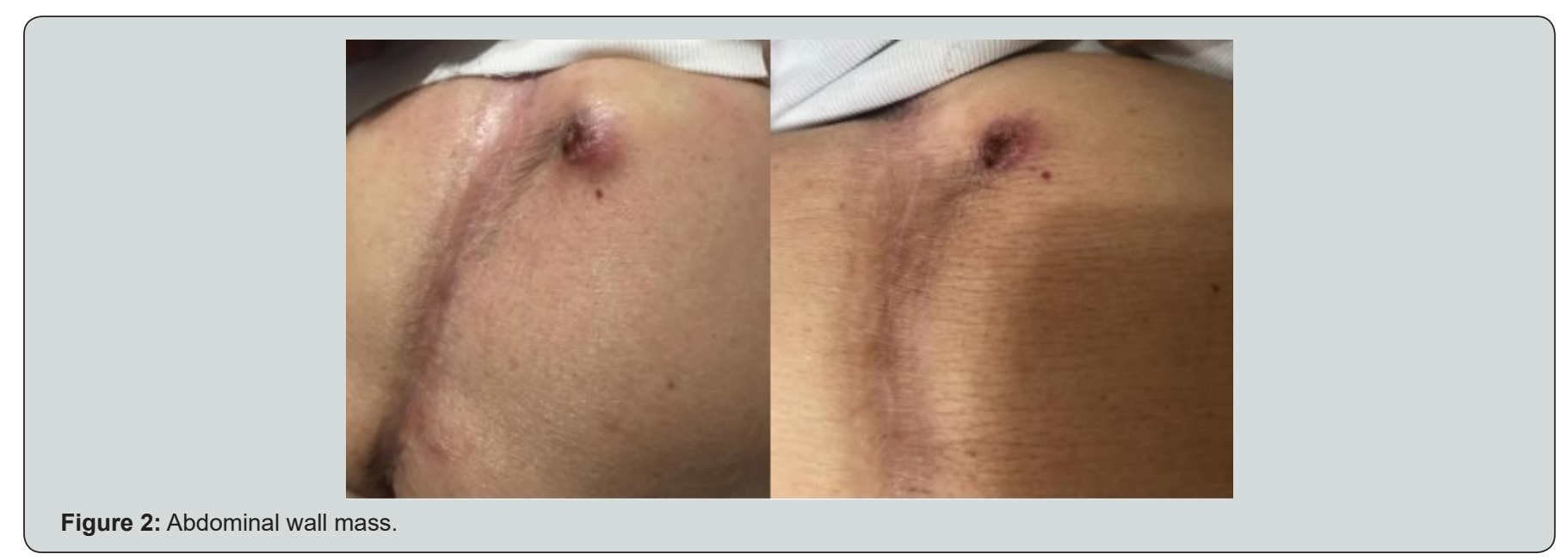

Figure 2: Abdominal wall mass

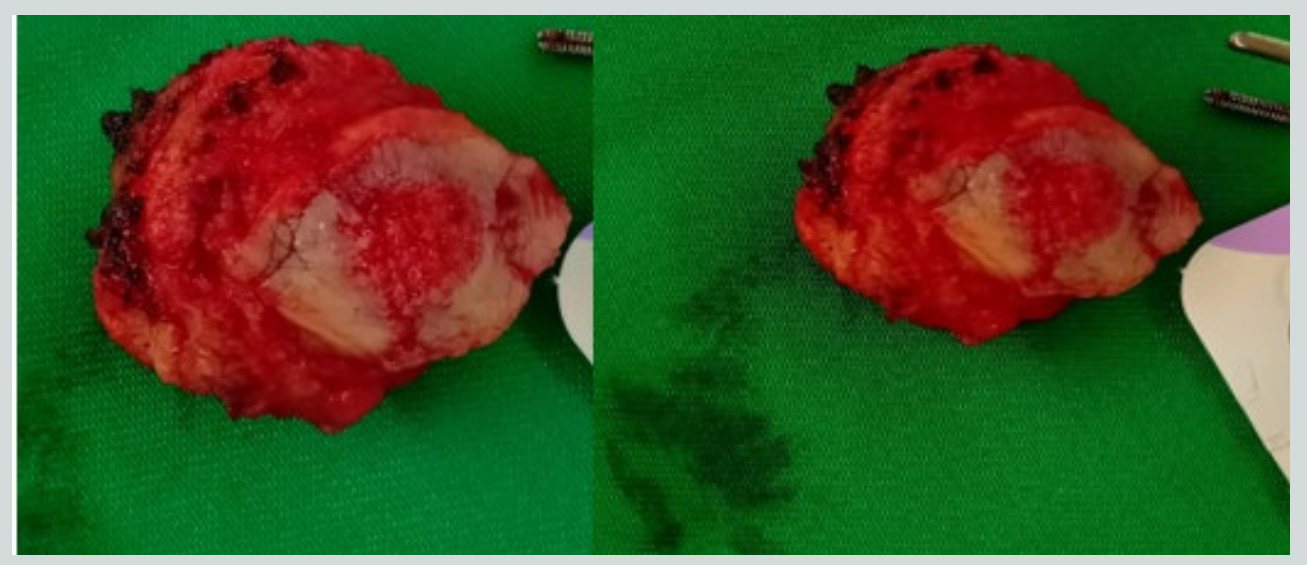

Figure 3: Excision of the mass.

He was followed up at 1, 3, 6 and 12 months with serial CT scan and serum tumor markers that revealed no local or distant recurrence. One and a half years after the operation, he presented with an abdominal wall mass that developed over the last 2 months. The physical examination revealed a hard-abdominal wall mass, lateral to the midline incision, approximately $3 \mathrm{~cm}$ in 
diameter, immobile and fixed with overlying skin ulceration as seen in Figure 2. The mass was then excised, and the histology revealed a metastatic deposit of a moderately differentiated adenocarcinoma consistent with the known primary gastric tumor (Figure 3).

One month later, FDG PET/CT was done and showed hypermetabolic activity in the liver and the para-aortic lymph nodes, suggestive of metastasis. Then, the patient continued the same chemotherapy.

\section{Discussion}

Skin metastasis refers to the abnormal development of cancer cells in the skin. It appears usually late after the diagnosis of the primary cancer [2]. Its overall prevalence is $5.3 \%$ of all visceral cancers [3]. In internal malignancies it is rarely the first sign (only in $0.8 \%$ ) [4] and in this case it is mostly linked to lung, kidney and ovary cancer [5]. Gastric cancer usually gives metastasis to the liver, peritoneal cavity and lymph nodes [2]. However, it can rarely give cutaneous metastasis and it appears usually in end stage disease and mostly on the chest, head and neck $[5,6]$.

Skin malignancy diagnosis depends primarily on biopsy and on immunohistological studies. An epithelial origin usually expresses cytokeratin on immunohistochemical study. These cytokeratins refers to the original tumor thus it is the most accurate to differentiate between primary and metastatic malignancy. Once we diagnose a metastatic malignancy, we should rule out other malignancies by specific test and imaging studies. A full body CT scan is required followed by ultrasound. CT had better sensitivity in detecting celiac lymph node, liver and lung metastasis, in contrast supraclavicular lymph node metastasis was better detected using US than using CT alone with a rate of 85 versus $28 \%$ [7]. Thus, the combination of US neck and CT neck/thorax/abdomen is most cost efficient and best for staging investigation [8].

A retrospective study showed the importance of FDG-PET for diagnosing the recurrence of a treated gastric cancer, it showed that FDG-PET had a specifity of $69 \%$ and a sensitivity of $70 \%$ [9]. Therefore, due to its low negative predictive value and low sensitivity, it shouldn't be used for the screening after the follow-up [10]. However, FDG-PET is useful for the prognosis of recurrent gastric cancer [10].

The treatment of skin malignancy depends on size, location, stage and type of the tumor and on the patient's general health and immune state and the risk/benefit ratio. Eight types of treatment are available: Surgery, radiation, chemotherapy, photodynamic therapy, immune therapy, targeted therapy, chemical peel and new studies on drug therapies.

Surgery by simple excision followed by radiotherapy or chemotherapy is the most used to treat gastric cancer with cutaneous metastatic patients [2]. However, in an advanced gastric cancer and when excision cannot be done, a combination of immunotherapy, molecular-targeted therapy and neoadjuvant chemotherapy was mostly used [10]. Unfortunately, chemotherapy isn't useful in the complete cure of gastric cancer, but it can increase survival rate, alleviate the symptoms and reduce the size of the tumor [11].

A meta-analysis showed highest survival rates with 3 drugs regimen containing $\mathrm{FU}$, an anthracycline, and cisplatin [12]. It also proved that regimens with bolus FU caused more toxicities than those with continuous infusion of FU, such as epirubicin, cisplatin [13]. New researchers are studying the benefit of these drugs before surgery to shrink tumor [1].

A recent study showed that high level of serum IL8 were associated with low chemotherapy response but the level of IL6 didn't have any effect [14].

A retrospective study of 4020 patients with cutaneous metastasis showed a low survival rate (1-34 months) depending on the primary cancer [8] For example, a cutaneous metastasis from the breast have a better prognosis than other cutaneous metastasis with internal primary origin [12]. Stomach cancer with cutaneous metastasis is among cancers with poor prognosis and was proved in a study of 200 cases with cutaneous metastasis that had a survival rate of 1.2 month for gastric cancer and none lived more than 9 years [15].

An opposing fact of 2 case reports of 2 patients (60 years and 65 years) with metachronous skin metastasis from gastric cancer and with no other metastasis, showed respectively a survival rate of more than 7.5 years and more than 3 year for each patient $[16,17]$.

\section{Conclusion}

Although the patient was metastasis free and with only one positive lymph node after gastrectomy, he presented after 1.5 year with skin metastasis, one of the rarest metastatic growth of gastric cancer. Therefore, it is recommended to be aware of any skin lesion specially in patients with history of cancer and take biopsy when there's suspicion to rule out metastasis.

\section{References}

1. Cesaretti M, Malerba M, Basso V, Boccardo C, Santoni R, et al. (2014) Cutaneous metastasis from primary gastric cancer: a case report and review of the literature. Cutis 93(4): E9-E13.

2. Hussein MR (2010) Skin metastasis: a pathologist's perspective. J Cutan Pathol 37(9): e1-e20.

3. Krathen RA, Orengo IF, Rosen T (2003) Cutaneous metastasis: a metaanalysis of data. South Med J 96(2): 164-168.

4. Lookingbill DP, Spangler N, Sexton FM (1990) Skin involvement as the presenting sign of internal carcinoma: a retrospective study of 7316 cancer patients. J Am Acad Dermatol 22(1): 19-26.

5. Schwartz RA (1995) Cutaneous metastatic disease. J Am Acad Dermatol 33(2): 161-185. 
6. Liu F, Yan WL, Liu H, Zhang M, Sang H (2015) Cutaneous metastases from gastric adenocarcinoma 15 years after curative gastrectomy. An Bras Dermatol 90(3): 46-50.

7. van Vliet EP, Steyerberg EW, Eijkemans MJ, Kuipers EJ, Siersema PD (2007) Detection of distant metastases in patients with oesophageal or gastric cardia cancer: a diagnostic decision analysis. Br J Cancer 97(7) 868

8. Lookingbill DP, Spangler N, Helm KF (1993) Cutaneous metastases in patients with metastatic carcinoma: a retrospective study of 4020 patients. J Am Acad Dermatol 29(2): 228-236.

9. De Potter T, Flamen P, Van Cutsem E, Penninckx F, Filez L, et al. (2002) Whole-body PET with FDG for the diagnosis of recurrent gastric cancer. Eur J Nucl Med Mol Imaging 29(4): 525-529.

10. Song Z, Wu Y, Yang J, Yang D, Fang X (2017) Progress in the treatment of advanced gastric cancer. Tumour Biol 39(7): 1010428317714626.

11. Wagner AD, Unverzagt S, Grothe W, Kleber G, Grothey A, et al. (2017) Chemotherapy for advanced gastric cancer. Cochrane Database Syst $\operatorname{Rev}(8)$.

12. Hu SC, Chen GS, Lu YW, Wu CS, Lan CC (2008) Cutaneous metastases from different internal malignancies: a clinical and prognostic appraisal. J Eur Acad Dermatol Venereol 22(6): 735-740.
13. Wagner AD, Grothe W, Haerting J, Kleber G, Grothey A, et al. (2006) Chemotherapy in advanced gastric cancer: a systematic review and meta-analysis based on aggregate data. J Clin Oncol 24(18): 29032909.

14. Zhai J, Shen J, Xie G, Wu J, He M, (2019) Cancer-associated fibroblastsderived IL-8 mediates resistance to cisplatin in human gastric cancer. Cancer Lett 454: 37-43.

15. Schoenlaub P, Sarraux A, Grosshans E, Heid E, Cribier B (2001) Survival after cutaneous metastasis: a study of 200 cases. Ann Dermatol Venereol 128(12): 1310-1315.

16. Früh M, Ruhstaller T, Neuweiler J, Cerny T (2005) Resection of Skin Metastases from Gastric Carcinoma with Long-Term Follow-Up: An Unusual Clinical Presentation. Onkologie 28(1): 38-40.

17. Morita J, Aoyama T, Amano S, Maezawa Y, Sawazaki S, et al. (2017) A case of long-term survival of cutaneous metastasis from primary gastric cancer. Gan To Kagaku Ryoho 44(12): 1393-1395.

\section{Your next submission with Juniper Publishers will reach you the below assets}

- Quality Editorial service

- Swift Peer Review

- Reprints availability

- E-prints Service

- Manuscript Podcast for convenient understanding

- Global attainment for your research

- Manuscript accessibility in different formats

(Pdf, E-pub, Full Text, Audio)

- Unceasing customer service

Track the below URL for one-step submission https://juniperpublishers.com/online-submission.php 